\title{
Evaluation of Coriander Germplasm for Green Leaf Purpose and Quality Parameters in Summer under Shadenet Conditions
}

\author{
G. Harshavardhan", S. Suryakumari, R.V.S.K. Reddy, D.R. Salomi Suneetha, \\ R.V. Sujatha and K. Giridhar
}

Dr. Y.S.R. Horticultural University, Horticulture College and Research Institute, Venkataramannagudem, West Godavari-534101, Andhra Pradesh, India

*Corresponding author

\begin{tabular}{|l|l}
\hline \multicolumn{1}{c|}{ A B S T R A C T } \\
\cline { 2 - 3 } \multicolumn{1}{l|}{$\begin{array}{l}\text { Keywords } \\
\text { Genotypes, LCC. }\end{array}$} & $\begin{array}{l}\text { Coriander is considered both as herb and spice. The experiment was conducted at } \\
\text { Horticultural College and Research Institute, Venkataramannagudem during } \\
\text { summer } 2015 \text { under } 50 \text { percent green colour shadenet with } 66 \text { coriander genotypes } \\
\text { and four checks to select an ideal plant type for green leaf purpose with desirable } \\
\text { growth, yield and quality parameters (Chlorophyll content and Vitamin C) which } \\
\text { are significantly superior to checks sadhana, sudha, suguna, LCC-234. Compared } \\
\text { to other entries evaluated the genotype LCC-176 recorded maximum yield } \\
\text { Article Info }\end{array}$ \\
$\begin{array}{ll}\text { Accepted: } \\
\text { 17 September 2017 } \\
\text { Available Online: } \\
\text { 10 November 2017 }\end{array}$ & $\begin{array}{l}\text { suitable for green foliage in coriander with desirable traits have been identified for } \\
\text { off season cultivation in summer under shadenet conditions. However, it is better } \\
\text { to grow in shadenet in summer for their better performance. }\end{array}$ \\
\hline \hline
\end{tabular}

\section{Introduction}

Coriander, Coriandrum sativum is a tropical and sub-tropical crop. The word coriander derived from the Greek name for bug, 'korion' (Diederichsen, 1996). Coriander is considered both as an herb and spice. When the aromatic plant is consumed fresh, it is considered as herb. When consumed fresh, it is called cilantro, green coriander, and Chinese parsley. Its grains are used as spice Leaves are particularly rich in vitamin $\mathrm{A}$ equivalent (42\%) $337 \mu \mathrm{g}$, vitamin C (33\%) 27 mg and vitamin $\mathrm{K} \quad 310 \mu \mathrm{g}$. Germplasm collection with good variability for the desirable characters is the basic requirement of any crop improvement programme
(Singahania et al., 2006). Coriander has great demand in summer. Hence the present investigation was taken up to observe the performances of coriander genotypes and select the promising genotypes for foliage yield in summer under shadenet conditions.

\section{Materials and Methods}

The experiment was laid out in an augmented block design with four checks repeated randomly in each block. The plot size was 1 m x 1 m. Fertilizers were applied @ 80 kg N, $35 \mathrm{~kg} \mathrm{P}, 60 \mathrm{~kg} \mathrm{~K}$ and $20 \mathrm{~kg} \mathrm{~S}$ and 10 ton cowdung per hectare (Anon., 2001). The 
entire amount of cowdung, phosphorus and potassium, with one-half of nitrogen from urea were applied during final land preparation. The rest of the nitrogen was topdressed at 30 days after sowing. The seeds (fruits) were rubbed for separating the two mericarps (seeds) which were soaked in water for 24 hours to enhance germination. Seeds were also treated with Bavistin at $2 \mathrm{~g}$ per $\mathrm{kg}$ of seeds prior to sowing in raised seed bed at $10 \mathrm{~cm}$ apart rows continuously by hand @ 30 $\mathrm{kg} / \mathrm{ha}$. Seeds were mixed with some loose soil (about four to five times of weight of seeds) to allow uniform sowing in rows and were covered with good pulverized soil just after sowing with gently pressed by hands. The sowing was done on 18 May 2015. Light watering was done to supply sufficient moisture needed for quick germination. Weeding and thinning was not done. For good germination, water was given to the plots two times per day with rose can till germination, later on one irrigation was applied. Harvesting of green foliage was done before bolting by cutting just beneath the soil with root intact after 40-45 days of sowing depending upon genotypes. Data was collected from the inner rows of each plot to avoid the border effect. In each unit plot, 5 plants were selected randomly for recording data on plant height. Number of leaves, leaf area, fresh leaf weight, foliage yield, chlorophyll content, vitamin C. the plot yield was converted to hectare yield. The collected data were properly analyzed statistically by Crop Stat Version7.2. The mean comparision was done at $5 \%$ level of probability.

\section{Results and Discussion}

The results presented in the Table 1 revealed positive changes in crop growth and yield compared to the checks evaluated, plant height at harvest ranged from 8.16 to 22.51 $\mathrm{cm}$. The highest plant height was recorded by LCC-7 $(22.51 \mathrm{~cm})$ which was superior to all other entries under evaluation. The minimum plant height at harvest was observed in LCC$117(8.16 \mathrm{~cm})$. Varied response of genotypes to environments in coriander plant height was reported by Giridhar and Sarada (2005).

LCC-9 (19.57) recorded significantly more number of leaves than any other entry evaluated. Our results are in concordance with those of Prabhu and Balakrishnamurthy (2006) from India and Moniruzzaman et al., (2013).

Twelve entries recorded significantly higher leaf area over the best check Sadhana (23.24 $\left.\mathrm{cm}^{2}\right)$. These entries are LCC-69 $\left(27.74 \mathrm{~cm}^{2}\right)$, LCC-154 $\left(29.45 \mathrm{~cm}^{2}\right)$, LCC-56 $\left(29.72 \mathrm{~cm}^{2}\right)$, AD-1 (30.21 cm $\left.\mathrm{cm}^{2}\right)$ LCC-190 (30.28 cm $\mathrm{cm}^{2}$, LCC-171 $\left(30.97 \mathrm{~cm}^{2}\right)$, LCC-50 $\left(31.02 \mathrm{~cm}^{2}\right)$, LCC-172 $\left(32.27 \mathrm{~cm}^{2}\right)$, LCC-188 $\left(32.65 \mathrm{~cm}^{2}\right)$, LCC-119 $\left(33.22 \mathrm{~cm}^{2}\right)$, LCC-47 $\left(33.62 \mathrm{~cm}^{2}\right)$ and LCC-185 $\left(33.75 \mathrm{~cm}^{2}\right)$, The minimum leaf area was observed in LCC-220 $\left(6.99 \mathrm{~cm}^{2}\right)$ which was significantly inferior to all the checks evaluated, similar studies were done and reported by Meena et al., (2014). The entries LCC-171 (4.72 $\mathrm{g}$ plant $\left.{ }^{-1}\right)$, LCC-176 (2.47 $\left.\mathrm{g} \mathrm{plant}^{-1}\right)$, LCC-175 (2.13 $\left.\mathrm{g} \mathrm{plant}^{-1}\right)$, LCC-69 (1.99 $\left.\mathrm{g} \mathrm{plant}^{-1}\right)$ and LCC-182 (1.89 g plant $\left.^{-1}\right)$ recorded significantly higher fresh leaf weight over the best check Sadhana (1.34 g plant $\left.^{-1}\right)$. Palanikumar et al., (2012) also reported that significant variation in fresh plant weight among the genotypes.

Eighteen entries recorded significantly higher yield over the best check Sadhana (5.218 t $\left.\mathrm{ha}^{-1}\right)$. These genotypes are LCC-176 (10.54 t $\left.\mathrm{ha}^{-1}\right)$, LCC-171 (9.743 $\left.\mathrm{t} \mathrm{ha}^{-1}\right)$, LCC-175 (9.443 $\left.\mathrm{t} \mathrm{ha}^{-1}\right)$, LCC-188 (9.018 $\left.\mathrm{t} \mathrm{ha}^{-1}\right)$, LCC$172\left(8.743 \mathrm{t} \mathrm{ha}^{-1}\right)$, LCC-119 (8.618 $\left.\mathrm{t} \mathrm{ha}^{-1}\right)$, LCC-190 (8.618 $\left.\mathrm{t} \mathrm{ha}^{-1}\right)$, LCC-154 (8.218 t $\mathrm{ha}^{-1}$ ), LCC-189 (8.118 t ha $\left.{ }^{-1}\right)$, LCC-69 (7.468 $\left.\mathrm{t} \mathrm{ha}^{-1}\right), \mathrm{LCC}-182\left(7.443 \mathrm{t} \mathrm{ha}^{-1}\right), \mathrm{LCC}-1$ (7.168 $\left.\mathrm{t} \mathrm{ha}^{-1}\right)$, LCC -194 (7.118 $\left.\mathrm{t} \mathrm{ha}^{-1}\right)$, LCC-185 (7.118 t ha $\left.{ }^{-1}\right)$, LCC-7 (7.068 t ha $\left.{ }^{-1}\right)$, LCC-60 (6.768 $\left.\mathrm{t} \mathrm{ha}^{-1}\right)$, LCC-47 (6.768 $\left.\mathrm{t} \mathrm{ha}^{-1}\right)$ and LCC-183 (6.743 t ha-1). 
Table.1 Desirable characters of coriander germplasm in summer under shadenet conditions

\begin{tabular}{|c|c|c|c|c|c|c|c|c|}
\hline S.No. & Genotype & $\begin{array}{l}\text { PH } \\
(\mathbf{c m})\end{array}$ & NL & $\mathrm{LA}\left(\mathrm{cm}^{2}\right)$ & FLW(g) & $\begin{array}{c}\text { Yield } \\
\text { (tonnes) }\end{array}$ & $\begin{array}{c}\text { CHL } \\
(\mathrm{mg} / \mathrm{g})\end{array}$ & $\begin{array}{l}\text { VITC } \\
(\mathrm{mg} / 100 \mathrm{~g})\end{array}$ \\
\hline 1. & $\mathrm{LCC}-1$ & 18.91 & 14.57 & 28.48 & 1.71 & 7.16 & 1.36 & 176.10 \\
\hline 2. & $\mathrm{LCC}-3$ & 18.41 & 14.57 & 24.71 & 1.55 & 6.36 & 2.31 & 196.10 \\
\hline 3. & $\mathrm{LCC}-6$ & 17.21 & 10.57 & 23.98 & 1.44 & 5.66 & 2.19 & 176.10 \\
\hline 4. & $\mathrm{LCC}-7$ & 22.51 & 7.57 & 27.67 & 1.84 & 7.06 & 2.91 & 146.10 \\
\hline 5. & $\mathrm{LCC}-9$ & 17.41 & 19.57 & 26.28 & 1.61 & 6.26 & 3.19 & 156.10 \\
\hline 6. & LCC -11 & 15.01 & 15.57 & 25.88 & 1.46 & 5.76 & 2.51 & 226.10 \\
\hline 7. & $\mathrm{LCC}-13$ & 11.96 & 8.32 & 20.97 & 0.85 & 3.51 & 3.61 & 136.10 \\
\hline 8. & LCC -17 & 11.06 & 6.32 & 23.07 & 0.95 & 3.91 & 2.88 & 176.10 \\
\hline 9. & $\mathrm{LCC}-19$ & 13.66 & 9.32 & 19.43 & 0.87 & 3.21 & 3.58 & 166.10 \\
\hline 10. & $\mathrm{LCC}-24$ & 12.86 & 10.32 & 20.67 & 1.04 & 3.81 & 3.69 & 156.10 \\
\hline 11. & $\mathrm{LCC}-30$ & 11.66 & 4.32 & 22.37 & 1.07 & 3.91 & 4.57 & 146.10 \\
\hline 12. & $\mathrm{LCC}-31$ & 12.56 & 9.32 & 21.63 & 0.83 & 3.21 & 3.27 & 136.10 \\
\hline 13. & $\mathrm{LCC}-32$ & 14.31 & 9.32 & 23.05 & 1.34 & 5.64 & 1.76 & 126.10 \\
\hline 14. & LCC -36 & 16.31 & 7.32 & 21.35 & 1.19 & 4.74 & 2.15 & 116.10 \\
\hline 15. & LCC - 37 & 11.41 & 7.32 & 22.18 & 1.23 & 4.94 & 3.26 & 156.10 \\
\hline 16. & LCC -38 & 12.71 & 6.32 & 16.32 & 1.08 & 4.04 & 2.25 & 196.10 \\
\hline 17. & $\mathrm{LCC}-43$ & 12.31 & 7.32 & 20.55 & 1.31 & 5.04 & 3.39 & 146.10 \\
\hline 18. & LCC - 44 & 11.01 & 7.32 & 16.82 & 1.14 & 4.24 & 2.92 & 146.10 \\
\hline 19. & LCC -47 & 16.81 & 6.57 & 33.62 & 1.55 & 6.76 & 2.47 & 106.10 \\
\hline 20. & LCC -49 & 15.51 & 8.57 & 25.75 & 1.22 & 4.96 & 2.49 & 156.10 \\
\hline 21. & LCC -50 & 16.81 & 8.57 & 31.02 & 1.43 & 5.76 & 3.62 & 206.10 \\
\hline 22. & LCC -53 & 12.41 & 7.57 & 27.25 & 1.16 & 4.96 & 3.93 & 156.10 \\
\hline 23. & LCC -56 & 16.31 & 7.57 & 29.72 & 1.33 & 5.36 & 3.47 & 206.10 \\
\hline 24. & LCC - 59 & 15.01 & 7.57 & 27.68 & 1.18 & 4.66 & 3.77 & 206.10 \\
\hline 25. & LCC -60 & 16.94 & 8.82 & 26.40 & 1.76 & 6.76 & 3.91 & 178.60 \\
\hline 26. & $\mathrm{LCC}-67$ & 13.64 & 6.82 & 21.90 & 1.39 & 6.16 & 2.43 & 218.60 \\
\hline 27. & LCC -69 & 16.84 & 9.82 & 29.17 & 1.99 & 7.46 & 3.65 & 218.60 \\
\hline 28. & $\mathrm{LCC}-70$ & 11.84 & 5.82 & 17.03 & 1.05 & 4.66 & 2.98 & 198.60 \\
\hline 29. & LCC -79 & 10.54 & 5.82 & 16.23 & 0.71 & 3.96 & 3.75 & 188.60 \\
\hline 30. & LCC -80 & 10.04 & 4.82 & 17.30 & 0.91 & 4.56 & 3.59 & 188.60 \\
\hline 31. & LCC -81 & 14.26 & 7.57 & 15.72 & 1.00 & 4.04 & 3.60 & 193.60 \\
\hline 32. & LCC -86 & 12.06 & 9.57 & 15.46 & 0.44 & 1.54 & 3.17 & 193.60 \\
\hline 33. & LCC -90 & 12.96 & 7.57 & 11.66 & 0.47 & 2.64 & 4.05 & 143.60 \\
\hline 34. & LCC -91 & 8.76 & 7.57 & 16.42 & 0.94 & 2.64 & 3.28 & 193.60 \\
\hline 35. & LCC - 112 & 9.16 & 6.57 & 16.59 & 1.16 & 4.34 & 2.60 & 143.60 \\
\hline 36. & LCC - 117 & 8.16 & 7.57 & 12.66 & 1.07 & 3.74 & 2.62 & 193.60 \\
\hline 37. & LCC - 119 & 15.16 & 10.57 & 33.22 & 1.80 & 8.61 & 2.50 & 168.60 \\
\hline 38. & LCC - 134 & 10.96 & 2.57 & 21.42 & 1.07 & 5.41 & 3.93 & 168.60 \\
\hline 39. & LCC -137 & 12.86 & 7.57 & 16.15 & 1.05 & 5.51 & 2.98 & 218.60 \\
\hline 40. & LCC - 154 & 14.96 & 15.57 & 29.45 & 1.69 & 8.21 & 4.38 & 168.60 \\
\hline 41. & LCC -156 & 14.36 & 9.57 & 26.68 & 1.19 & 5.71 & 3.17 & 168.60 \\
\hline
\end{tabular}




\begin{tabular}{|c|c|c|c|c|c|c|c|c|}
\hline 42. & LCC -169 & 10.96 & 10.57 & 19.22 & 1.13 & 4.81 & 3.11 & 218.60 \\
\hline 43. & LCC -171 & 10.56 & 11.07 & 30.97 & 4.72 & 9.74 & 3.69 & 131.10 \\
\hline 44. & LCC -172 & 11.56 & 9.07 & 32.27 & 1.76 & 8.74 & 3.05 & 181.10 \\
\hline 45. & LCC -175 & 10.46 & 4.07 & 25.80 & 2.13 & 9.44 & 3.90 & 131.10 \\
\hline 46. & LCC -176 & 11.96 & 10.07 & 26.63 & 2.47 & 10.54 & 2.38 & 231.10 \\
\hline 47. & LCC -182 & 11.76 & 8.07 & 24.70 & 1.89 & 7.44 & 2.76 & 181.10 \\
\hline 48. & LCC -183 & 10.56 & 12.07 & 23.97 & 1.74 & 6.74 & 3.73 & 231.10 \\
\hline 49. & LCC -185 & 11.69 & 10.07 & 33.75 & 1.31 & 7.11 & 3.64 & 106.10 \\
\hline 50. & LCC -188 & 14.79 & 7.07 & 32.65 & 1.69 & 9.01 & 2.19 & 306.10 \\
\hline 51. & LCC -189 & 13.09 & 8.07 & 26.75 & 1.56 & 8.11 & 3.30 & 256.10 \\
\hline 52. & LCC -190 & 12.99 & 6.07 & 30.28 & 1.54 & 8.61 & 3.18 & 206.10 \\
\hline 53. & LCC -194 & 11.89 & 9.07 & 27.45 & 1.12 & 7.11 & 1.82 & 256.10 \\
\hline 54. & LCC -204 & 16.89 & 10.07 & 28.72 & 1.47 & 6.21 & 4.15 & 156.10 \\
\hline 55. & LCC -209 & 11.74 & 5.57 & 12.82 & 1.27 & 4.21 & 3.02 & 131.10 \\
\hline 56. & LCC -211 & 13.74 & 4.57 & 18.66 & 1.25 & 4.71 & 2.86 & 171.10 \\
\hline 57. & LCC -218 & 11.94 & 5.57 & 12.19 & 0.96 & 3.41 & 3.73 & 181.10 \\
\hline 58. & LCC -220 & 9.34 & 6.57 & 6.99 & 1.04 & 4.31 & 3.08 & 171.10 \\
\hline 59. & LCC -221 & 12.04 & 5.57 & 13.03 & 1.00 & 3.81 & 4.19 & 121.10 \\
\hline 60. & LCC -228 & 11.14 & 7.57 & 8.49 & 1.08 & 4.01 & 4.51 & 131.10 \\
\hline 61. & LCC -237 & 12.54 & 8.57 & 21.64 & 1.60 & 5.69 & 3.79 & 236.10 \\
\hline 62. & LCC -238 & 14.44 & 9.57 & 25.27 & 1.65 & 5.99 & 2.87 & 156.10 \\
\hline 63. & LCC -277 & 10.44 & 7.57 & 10.88 & 1.74 & 5.59 & 3.27 & 156.10 \\
\hline 64. & LCC -298 & 15.14 & 6.57 & 28.44 & 1.33 & 4.19 & 2.09 & 196.10 \\
\hline 65. & $\mathrm{AD}-1$ & 15.44 & 7.57 & 30.21 & 1.29 & 4.19 & 3.83 & 216.10 \\
\hline 66. & Swathi & 12.84 & 8.57 & 15.14 & 1.15 & 4.09 & 3.25 & 196.10 \\
\hline 67. & Sadhana(C) & 14.11 & 8.64 & 23.24 & 1.34 & 5.21 & 3.66 & 188.20 \\
\hline 68. & Sudha(C) & 13.06 & 8.55 & 20.71 & 1.12 & 4.44 & 3.32 & 179.10 \\
\hline 69. & Suguna $(\mathrm{C})$ & 13.26 & 8.55 & 22.39 & 1.24 & 5.10 & 3.45 & 178.20 \\
\hline \multirow[t]{4}{*}{70.} & LCC $-234(\mathrm{C})$ & 12.72 & 8.55 & 19.40 & 1.15 & 4.70 & 3.17 & 179.10 \\
\hline & Mean & 13.30 & 8.42 & 22.46 & 1.35 & 5.48 & 3.19 & 170.92 \\
\hline & L.S.D. (5\%) & 2.29 & 2.05 & 5.51 & 0.33 & 1.29 & 1.21 & 5.87 \\
\hline & CV (\%) & 11.95 & 16.61 & 17.81 & 18.67 & 18.3 & 24.6 & 22.45 \\
\hline
\end{tabular}

Note: PH-Plant height, NL-Number of leaves, LA-Leaf area, FLW-Fresh leaf weight, Y-Yield, CHL-Chlorophyll content, VIT C- Vitamin C.

Minimum yield was observed in the genotype LCC-86 $\left(1.543 \mathrm{tha}^{-1}\right)$, the present findings are in accordance with the reports of Palanikumar et al., (2012) and Moniruzzaman et al., (2013).

The entries LCC-30 (4.57 $\left.\mathrm{mg} \mathrm{g}^{-1}\right)$ and LCC$228\left(4.51 \mathrm{mg} \mathrm{g}^{-1}\right)$ recorded higher chlorophyll content over the best check Sadhana (3.66 mg $\left.\mathrm{g}^{-1}\right)$. The lowest chlorophyll content was observed in the entry LCC-1 $\left(1.36 \mathrm{mg} \mathrm{g}^{-1}\right)$.
Ben et al., (2014) reported that coriander genotypes differed significantly in their leaf chlorophyll content. These findings are also corroborates with the findings of Kamineni $e t$ al., (2008) and Naidu (2011).

Twenty one genotypes recorded significantly higher vitamin $\mathrm{C}$ content than the best check Sadhana $\left(180.20 \mathrm{mg} 100 \mathrm{~g}^{-1}\right)$. The best ten elite entries are LCC-188 (306.20mg $\left.100 \mathrm{~g}^{-1}\right)$, LCC-189 (256.10mg $\left.100 \mathrm{~g}^{-1}\right)$, LCC -194 
(256.10mg $\left.100 \mathrm{~g}^{-1}\right)$, LCC-237 (236.10 mg $\left.100 \mathrm{~g}^{-1}\right)$, LCC-176 (231.10 mg $\left.100 \mathrm{~g}^{-1}\right)$, LCC183 (231.10 mg $\left.100 \mathrm{~g}^{-1}\right)$, LCC-11 (226.10 mg $\left.100 \mathrm{~g}^{-1}\right)$, LCC-67 (218.60 mg $\left.100 \mathrm{~g}^{-1}\right)$, LCC69 (218.60 mg $\left.100 \mathrm{~g}^{-1}\right)$ and LCC-137 (218.60 $\left.\mathrm{mg} 100 \mathrm{~g}^{-1}\right)$. The lowest vitamin $\mathrm{C}$ content was observed in LCC-47 (100.60 mg $\left.100 \mathrm{~g}^{-1}\right)$. Similar studies were reported by Lee and Kader (2000) and Vallejo et al., (2003).

\section{Acknowledgement}

I am very much thankful to my guide and members of Horticultural College and Research Institute, Venkataramannagudem, Dr. Y.S.R.H.U of their help and cooperation during the course of my research work. I am overwhelmed with gratitude to all my respondents, without whose whole hearted cooperation, this study would not have been fruitful. I extend my gratitude to the Dr. Y.S.R. Horticultural University, Venkataramannagudem for providing financial support in the form of stipend during my course of study.

Above all I am extremely thankful and grateful to God who has blessed me to be what I am today.

\section{References}

Anonymous. 2001. Annual Report for 20002001. Spices Research Centre, Shibganj, Bogra. p. 37.

Diederichsen, A. 1996. Coriander (Coriandrum sativum). International Plant Genetic Resources Institute (IPGRI), Rome, 245 pp.

Giridhar, K. and Sarada, C. 2005. Identification of coriander (Coriandrum sativum L.) genotypes for vertisols of Andhra Pradesh. National Symposium on Current Trends in Onion, Garlic, Chillies and Seed Spices-Production, Marketing and Utilization, SYMSAC-II,
25-27 November. NRCOG,

Rajgurunagar, pp.92.

International Rice Research Institute. 2007. Statistical procedures for agricultural research

Kamineni, L.N.R., Kalidasu, G. and Sarada, C. 2008. Drought tolerance of coriander (Coriandrum sativum Linn.) genotypes in rainfed vertisols. Envoinformatics, p. 73.

Lee, S.K. and Kader, A.A. 2000. Preharvest and postharvest factors influencing vitamin C content of horticultural crops. Postharvest biology and technology, 20 (3), pp. 207-220.

Meena, K.Y, Kale S.V. and Meena P.O. 2014. Correlation coefficient and path analysis in coriander. International Journal of Scientific and Research Publications. 4(6): 2250-3153.

Moniruzzaman, M., Rahman, M.M., Hossain, M.M., Karim, A.S. and Khaliq, Q.A. 2013. Evaluation of coriander (Coriandrum sativum L.) genotypes for foliage yield and its attributes. Bangladesh Journal of Agricultural Research, 38 (1), pp.175180.

Naidu, T. 2011. Evaluation of coriander genotypes for drought tolerance. Spices and Aromatic Plants: Status and Improvement, p.133.

Palanikumar, M., Rajamani, K. and Muthiah, A. 2012. Correlation studies in coriander (Coriandrum sativum L.) genotypes for fresh biomass yield and oil content under different seasons. Crop Res. 44 (1 \& 2): 217-221.

Prabhu, T. and Murthy, G. 2006. Evaluation of coriander (Coriandrum sativum L.) accessions under irrigated conditions for growth, yield and quality. Proc. Nat. Sem. Emerging Trends in Production, Quality, Processing and Export of Spices, 28-29 March, Coimbatore pp 13. Saxena, R.P, Pandey, V.P, Datta, J. and 
Gupta, R.K. 2005. Performance of coriander entries at Kumarganj, Faizabad. National Symposium on Current Trends in Onion, Garlic, Chillies and Seed Spices - Production and Utilization, SYMSAC-II, 25-27 November, $N R C O G$, Rajgurunagar, pp. 55-56.

Singhania, D.L., D. Singh and R. S. Raje. 2006. Coriader. In: Ravindran, P. R., K. N. Babu, K. N. Shiva. and J. A. Kallupurackal (eds.). Advances in
Spices and Achievments of Spices Research in India since Independence. Pp. 678-695.

Vallejo, F., Tomas-Barberan, F.A. and García-Viguera, C. 2003. Effect of climatic and sulphur fertilisation conditions, on phenolic compounds and vitamin $\mathrm{C}$, in the inflorescences of eight broccoli cultivars. European food research and technology, 216 (5), pp.395-401.

\section{How to cite this article:}

Harshavardhan, G., S. Suryakumari, R.V.S.K. Reddy, D.R. Salomi Suneetha, R.V. Sujatha and Giridhar, K. 2017. Evaluation of Coriander Germplasm for Green Leaf Purpose and Quality Parameters in Summer under Shadenet Conditions. Int.J.Curr.Microbiol.App.Sci. 6(11): 23992404. doi: https://doi.org/10.20546/ijcmas.2017.611.284 